\title{
Sediment contamination of an urban canal-a case study approach for an integrated assessment of organic sediment contamination
}

\author{
M. Ricking ${ }^{1,2}(\mathbb{D}) \cdot$ K. Frische ${ }^{3} \cdot$ J. Schwarzbauer ${ }^{3}$
}

Received: 25 March 2020 / Accepted: 23 July 2020 / Published online: 15 August 2020

(C) The Author(s) 2020

\begin{abstract}
Purpose The pesticide DDT and its metabolites represent a contamination risk for the aquatic environment, especially the polar metabolite DDA. The study provides a quantitative assessment of long-term pollution risks from sedimentary DDT residues with a special focus on DDA. It presents an overview of the contamination range of different DDX compounds in the sediments of a canal in Berlin (Germany), resulting from a former industrial point source that has implications for drinking water resources in the nearby area. The comprehensive analysis scheme provides information on free accessible and potentially metabolized precursors also in the non-extractable residues. This allows a quantitative assessment of the DDA pollution potential derived from the sedimentary DDT residues.

Materials and methods The area was investigated for fine-grained sediment by means of a geo-electric mapping. Twelve sediment cores were taken in four areas (three in each section). A wider range of precursor metabolites has been included due to their transformation potential to the polar metabolite DDA. The sediments were analysed quantitatively for extractable and easily releasable fractions by application of a variety of degradation techniques as well as a dispersion extraction procedure on the sediment samples. These extracts were fractionated and subsequently analysed by GC-MS.

Results and discussion Concentrations were obtained for extractable and bound metabolites. Different scenarios for the calculation of the amount of contaminated sediment are displayed as a tool for contamination assessment. The formation potential of DDA as the water-soluble metabolite is presented. Several precursor metabolites, e.g. DDD and DDMS, extractable from the sediment organic matter, revealed a high potential for a long-term formation of DDA, especially in the easily releasable fraction (via hydrolysis) with a mean concentration of up to $11,000 \mathrm{\mu g} \mathrm{g}^{-1}$ dry sediment. The resulting DDA contamination potential represents a significant pollution risk for the groundwater from a downstream waterworks area and by remobilisation into the whole ecosystem and adjacent rivers. Conclusions The application of the presented methods provides a tool for a quantitative assessment of the long-term release potential of DDA under different scenarios by a comprehensive analysis of contaminated sediments (and soils). This approach can be transferred to pollutants that are also characterized by a complex metabolism accompanied by bound residue formation.
\end{abstract}

Keywords DDX-related compounds $\cdot$ Sequential degradation $\cdot$ Non-extractable residues (NER) $\cdot$ DDA formation potential

\section{Responsible editor: Henner Hollert}

Electronic supplementary material The online version of this article (https://doi.org/10.1007/s11368-020-02738-5) contains supplementary material, which is available to authorized users.

M. Ricking

mathias.ricking@uba.de; ricking@zedat.fu-berlin.de

1 Environment Agency Germany, Wastewater Technology Research, Corrensplatz 1, 14195 Berlin, Germany

2 Freie Universität Berlin, Department of Earth Sciences, Malteserstr. 74-100, 12249 Berlin, Germany

3 Insitute of Geology and Geochemistry of Petroleum and Coal, RWTH Aachen University, Lochnerstr. 4-20,

52064 Aachen, Germany

\section{Introduction}

In sediments, organic pollutants can become incorporated by various ways into the organic matter and metabolized microbially. With respect to the aquatic environment, organic contaminants can be associated with the suspended particulate matter and released again by desorption processes (Northcott and Jones, 2000; Semple et al. 2003). Within the sedimentary organic matter (SOM), which consists amongst others of humic substances, an extractable and non-extractable fraction (NER) of pollutants can be differentiated and analysed separately by sequential degradation reactions (Eschenbach et al. 1994). Operationally defined, the extractable fraction 
represents the direct bio-available/bio-accessible contamination potential for the aquatic environment due to the weak bonding to the SOM by van der Waals forces or ionic attraction. The non-extractable fraction can be divided into reversible bound and irreversible bound residues, representing temporary or final depositions, respectively (see Section 2.4).

The chemical interactions of organic substances with sediments and soils (natural polymers or geo-sorbents) may result in strong binding and slow subsequent release rates that significantly affect remediation rates and endpoints (Scheunert and Schröder 1998). Hence, this is an important effect in particular for the risk assessment of pollutants. The underlying physical and chemical processes or phenomena potentially responsible for this apparent sequestration of persistent, bioaccumulative and toxic substances (PBTs) by geo-sorbents are only partially understood (Nowak et al. 2014; Kästner et al. 2016; Schäffer et al. 2018; Taylor et al. 2019). Recent observations suggest that $\mathrm{PBT}$ interactions with geo-sorbents comprise different organic and inorganic surfaces and matrices, and distinctions are clearly visible along these lines particularly with regard to the roles of inorganic micropores (e.g. in soot or clay minerals), natural organic matter components, combustion residue particulate carbon and spilled organic liquids. Definitely, SOM is not uniformly spread over all solid phases and not universally treatable as a well-defined organophilic phase with different $K_{\text {om }}$ values, as more than one order of magnitude difference in calculated distributions between $K_{\mathrm{om}}$ and solubility or $\log K_{\mathrm{ow}}$ are reported (Takada and Eganhouse 1998; Ehlers and Loibner, 2006). Partition-like interaction with a "microscopic non-polar organic environment", humic-membrane-like structure, polar hydrophobic exterior and non-polar hydrophobic interior is to be expected.

Detailed information related to the formation of nonextractable residues has been published by Luthy et al. (1997), Northcott and Jones, (2000), Schwarzbauer et al. (2003), Ehlers and Loibner (2006) and Kästner et al. (2016). The formation of NER in geo-polymers is realized through stronger ionic and covalent bonding, entrapment (inclusion in cavities) and biological processes. The reversibility of sorption-desorption is based on geochemical parameters like functional groups, quality of the $\mathrm{SOM}, \mathrm{pH}$, Eh and water chemistry. Important for the microbial degradation are the strength of the bonding and the degree of incorporation into the geo-polymers. Toxic effects are reduced due to limited bio-availability of bound pollutants resulting in a higher persistence and modified degradation fates relative to laboratory model systems (Garrison et al. 2014). Sequential chemical treatments are applied to selectively release compounds depending on the character of incorporation (Semple et al. 2003; Schwarzbauer et al. 2003; Kalathoor et al. 2015).

To address the complete contamination of riverine systems, the non-extractable fraction, characterized by incorporation due to covalent bonding, can reveal a long-term contamination risk due to their remobilisation potential of organic compounds to the environment. Especially, polar organic compounds exhibiting considerable solubility represent a contamination risk for the aquatic ecosystem.

According to Zhu et al. (2019), the degradation of DDT in the NER was differentiated into three categories (including the initial DDT) in order to clarify not only the further cleavage of the aromatic ring structure but also the degree of degradation by dehalogenation, functional group formation and loss of a $\mathrm{C}$-atom. Halogenated organic compounds are prone to a high environmental persistence. Pesticides such as DDT, exhibiting the main contamination revealed in this study, are well-known pollutants. They are characterized by high enrichment factors in sediment (Eganhouse and Pontolillo, 2008; Eganhouse et al. 2018, Taylor et al. 2019), resulting in a strong tendency for geo-accumulation, act as sinks and long-term sources for aromatic organic halogens (AOHs).

DDT has been applied worldwide between the 1940s and 1986 as an insecticide in agriculture and is still allowed for treatment of vectors such as malaria (WHO 2011). It is now classified as one of the "Dirty Dozen" according to the Stockholm Convention. DDT is also an intermediate in the formulation of Dicofol as an acaricide, miticide and antifouling treatment. Due to the high persistence and chronic toxicity, its use as a pesticide has been prohibited worldwide since the early 1970s worldwide. Even very recent publications from Asia (Babut et al. 2019) and Europe (Qu et al. 2019) report a high percentage of DDX (sum of all analysed DDT metabolites) in the line of organochlorine pesticides (OCPs). A recent review states the effort of DDX-removal from environmental samples is still a technical topic in the EU (Vagi and Petsas 2020).

The water solubilities of DDT, DDD and DDE at $25^{\circ} \mathrm{C}$ are $5.5 \mu \mathrm{g} \mathrm{L}^{-1}, 20.0 \mu \mathrm{g} \mathrm{L}^{-1}$ and $14 \mu \mathrm{g} \mathrm{L}^{-1}$, respectively. According to Lee et al. (1994), the $\log K_{\mathrm{ow}}$ values are 6.91, 6.22 and 6.96, respectively.

Environmental contamination by persistent organic compounds is referred to both point sources and diffusive sources. Point sources may be related to industrial production effluents, municipal emissions or agricultural activities. Diffusive sources may be surface run-off, deposition and atmospheric transport including long-range atmospheric transport (LRAT) which is the major pathway for the transfer of persistent pollutants to remote sites (Kallenborn 2006; Becker et al. 2008).

The global distribution of DDT and its metabolites has been studied intensively (e.g. Marei et al. 1978; de la Cal et al. 2008; Bettinetti et al. 2016; Mitra et al. 2019), but DDA has been neglected widely. Only a few studies considered this metabolite (Heberer and Dünnbier 1999; Schwarzbauer et al. 2003; Heim et al. 2005; Wan et al. 2005; Chen et al. 2012; Garrison et al. 2014), despite an early identification in 1945 (Strohlman and Smith 1945; White and Sweeny 1945). 
In the present study, subaquatic sediments of the Teltow Canal in Berlin (Germany) were intensively sampled and analysed close to a point source (see Fig. 2).

The map shows the Berlin area within the Elbe River Basin and a detailed view of the sampling area on the Teltow Canal with the point source at the eastern end of the canal. The factory (BC) discharged production wastewater from pesticides into the canal, where the Johannisthal waterworks some $6 \mathrm{~km}$ downstream produced drinking water.

The canal is used as a drainage for rainwater, industrial wastewater, wastewater treatment plants and as a shipping canal for industrial supply, with a length of approximately $38 \mathrm{~km}$ and a mean depth of $2 \mathrm{~m}$. It is characterized by a very slow flow velocity and discharge (WSA 2018, average discharge at the lock Kleinmachnow: $8.97 \mathrm{~m}^{3} \mathrm{~s}^{-1}$ ), occasional flow direction reversal, a high sedimentation rate in favoured accumulation areas and mostly anoxic conditions at the sediment-water interface. High accumulation rates and undisturbed deposition are present in these subaquatic sediments. The former industrial plant is located at the eastern border of the canal, close to the Dahme River. Between the 1940s and 1986 , about $60,000 \mathrm{t}$ of DDT were synthesized and formulated on site (Heim et al. 2005). Emission of untreated neutralised effluents leads to a strong contamination of canal sediment by DDT and other halogenated compounds for decades. Therefore, from 1995 to 1999, after removal of an underwater barrier, an excavation of approx. 180,000 t of contaminated sediment $\left(\sim 135,000 \mathrm{~m}^{3}\right)$ was realized, which led to long-term remobilisation processes (Heim et al. 2005; Frische et al. 2010). The production data are published in Heim et al. (2005).

A few kilometres downstream of the study area, a waterworks is located using riverbank filtration, posing a risk of water contamination by the DDT-metabolite DDA and all precursor metabolites (see Fig. 1) through remobilisation processes via professional shipping activity.

The aim of this study was to obtain not only an overview on the actual contamination range referring to the formation potential of water-soluble DDA (water solubility at $\mathrm{pH} 5-10$ is 20-59 $\mathrm{g} \mathrm{L}^{-1}$; Frische et al. 2010) from the remaining DDTcontaminated sediment of the canal but also the quantitative estimation of the sedimentary contamination release potential. The release behaviour of DDA under dynamical conditions was experimentally investigated to get a more precise insight into the environmental fate. Additionally, further information about the degradability/persistence of DDA is deduced (Fig. 2).

In the literature, the kinetics in biota (animals, humans) are described as fast; the environmental kinetics are mostly unknown.

DDT serves as a representative for compounds for other environmentally relevant compounds with similar toxicity, bio-accumulation potential, physicochemical features, degradability and formation potential of NER like methoxychlor and low molecular PAHs (Eschenbach et al. 2001).

\section{Materials and methods}

\subsection{Field mapping and sampling}

The determination of the sediment thickness was a prerequisite for targeted sediment sampling in the Teltow Canal and the information about the sediment structure and the distribution of organoleptic muds. With the help of geophysical measuring and interpretation methods, the spreading of muds in a $1.25-\mathrm{km}$ long section of the canal could be inquired. For this purpose, a combination of hydro acoustic measurements (echo sounder) and special methods of water geo-electric was applied.

\subsubsection{Preliminary remarks on echolocation}

The study concept is based on a combination of hydro acoustic measurements (dual-frequency sediment echo sounder) and geo-electrical water bottom mapping. Already during the measurements, it was found that the sediments in the study area were relatively "sound-reflecting" sediments with an unexpectedly hard sediment surface. The echolocation was carried out mainly to determine the depth of the water and for the evaluation of the sandy basis of the canal for the interpretation of the measurement data.

\subsubsection{Methodological basics}

The special process of water geo-electrics was realized through measuring cables that are towed directly above the sediment surface. From these results a high spatial resolution for the uppermost sediment layer regardless of the water depth, whose thickness can be determined with an accuracy of approx. $10 \%$.

As a physical principle, geo-electrics uses the different behaviour of substances with regard to their resistivity, in order to be able to withstand an approximate horizontal storage of the layers being able to determine material properties and spatial limitations. The specific electrical resistance of a layer is essentially determined by the following components:

- Solid sand, gravel grains: = infinitely high

- Pure clay: mostly under $10 \Omega \mathrm{m}^{-1}$

- Average waters $10 \ldots 40 \Omega \mathrm{m}^{-1}$

- Sediments with high organic content: sludge $\leq$ water

The resistance of a loose sediment depends on the water saturation of the water, and the particle size distribution and 


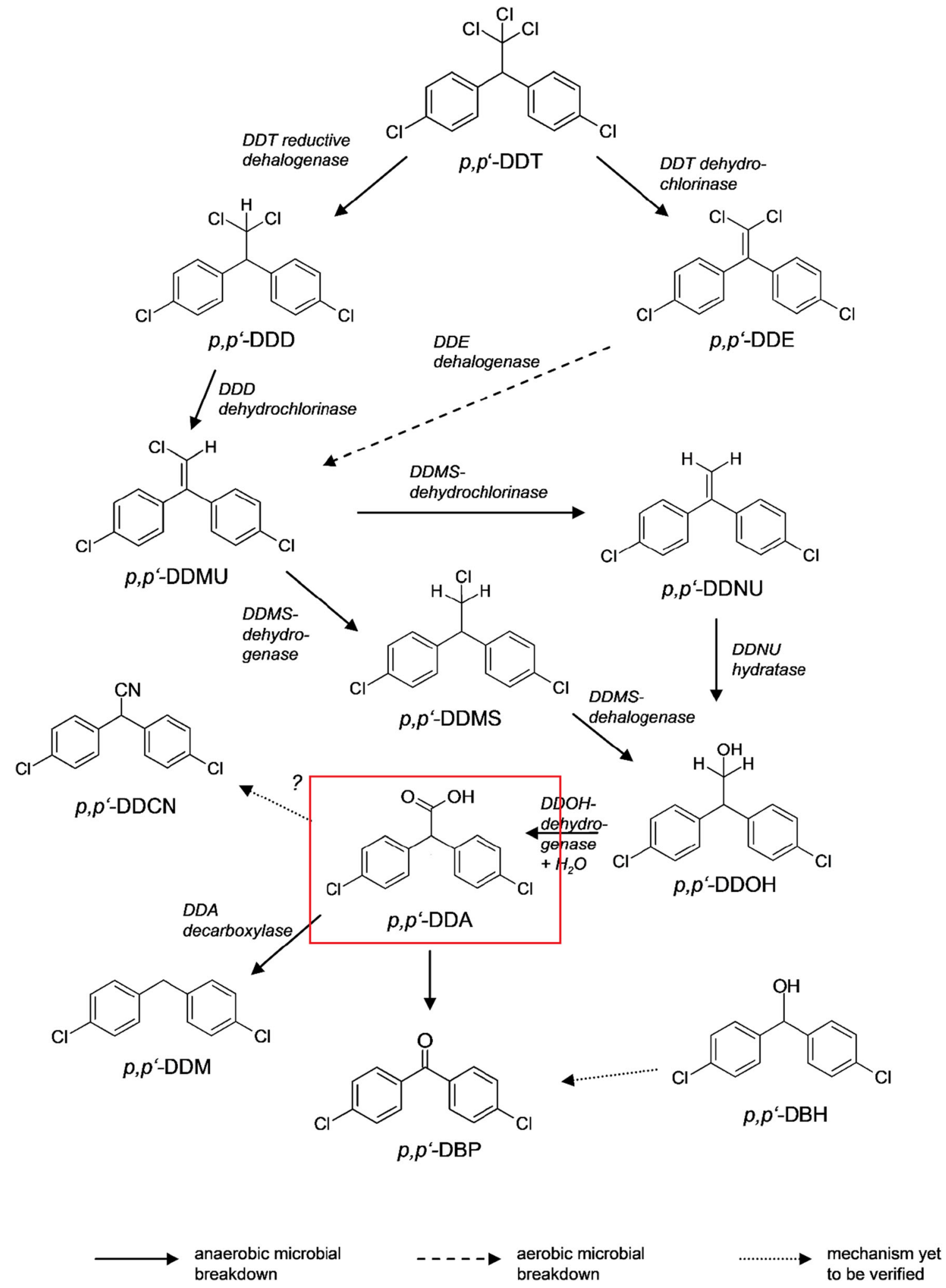

Fig. 1 Anaerobic metabolic pathway of DDT (modified) according to Wetterauer et al. (2012)

charged surfaces like clay minerals present in mud. This relationship forms the basis for a layer structure of the substrate.

The field mapping of the sediment area was performed by geo-electric measurements in cooperation with Inc. FUGRO.
Three profiles were taken along a stretch of $1250 \mathrm{~m}$. Distinctive areas in the sediment body are rich in humic matter with elevated TOC contents (see Fig. 4; see Table 1) with a geo-electric resistance of less than $35 \Omega \mathrm{m}^{-1}$ in the sediment 


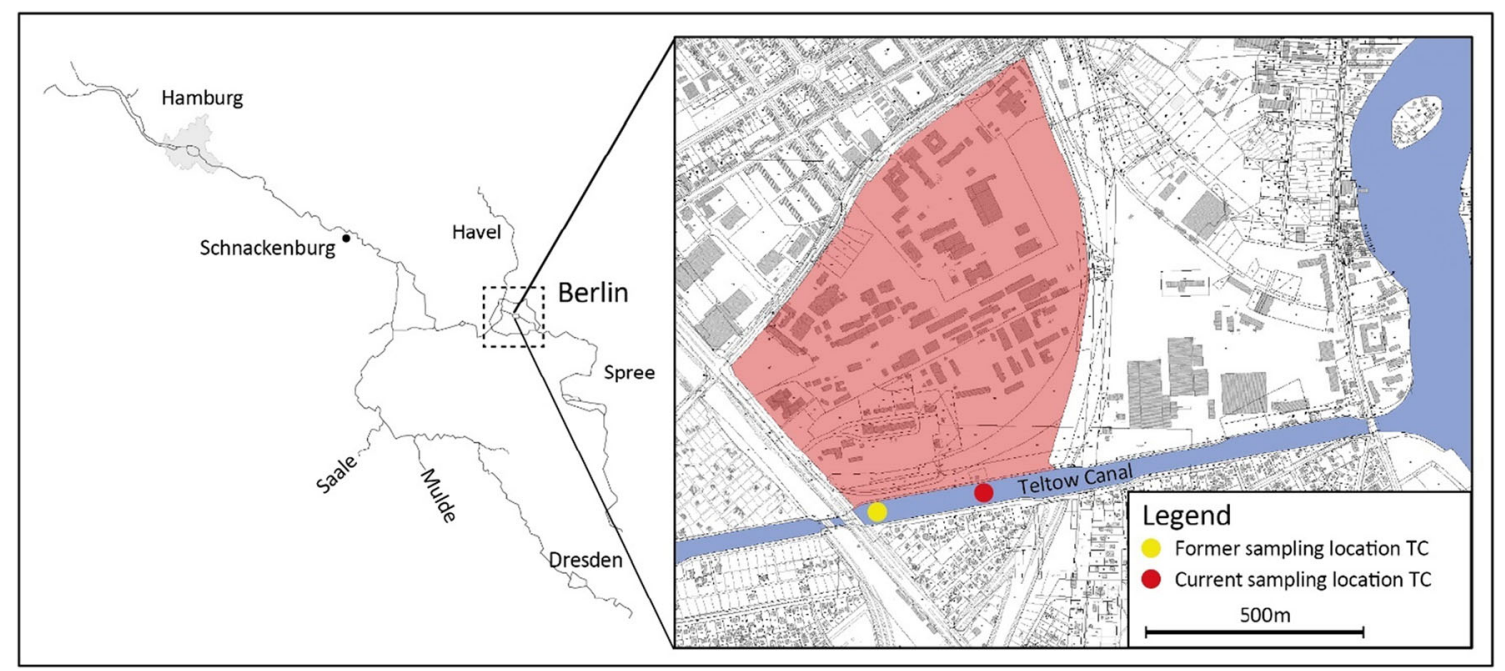

Fig. 2 Map of the Elbe River with an insert of the sampling area in Berlin, located at the eastern end of the Teltow Canal (detail view of the sampling area and the production site is marked in red)

Table 1 Lithology of the sediment cores with TOC content and bulk density

\begin{tabular}{|c|c|c|c|c|c|}
\hline Area & Core & Layer & Depth $(\mathrm{cm})$ & TOC (\%) & Bulk density $\left(\mathrm{g} \mathrm{cm}^{-3}\right)$ \\
\hline \multirow[t]{7}{*}{ D } & 1 & Top & $20-35$ & 9.29 & 0.70 \\
\hline & 1 & Middle & $35-45$ & 6.91 & 0.72 \\
\hline & 1 & Base & $45-60$ & 4.52 & 0.76 \\
\hline & 2 & Top & $09-26$ & 1.30 & 0.92 \\
\hline & 2 & Middle & $26-43$ & 0.86 & 0.83 \\
\hline & 3 & Middle & $26-44$ & 3.82 & 0.82 \\
\hline & 3 & Base & $44-62$ & 1.82 & 0.92 \\
\hline \multirow[t]{9}{*}{$\mathrm{C}$} & 4 & Top & $12-27$ & 9.46 & 0.67 \\
\hline & 4 & Middle & $27-44$ & 10.31 & 0.65 \\
\hline & 4 & Base & $44-59$ & 11.16 & 0.66 \\
\hline & 5 & Top & $11-26$ & 9.11 & 0.60 \\
\hline & 5 & Middle & $26-43$ & 6.12 & 0.71 \\
\hline & 5 & Base & $43-58$ & 3.33 & 0.82 \\
\hline & 6 & Top & $06-21$ & 7.53 & 0.60 \\
\hline & 6 & Middle & $21-49.5$ & 5.13 & 0.65 \\
\hline & 6 & Base & $49.5-64.5$ & 2.73 & 0.76 \\
\hline \multirow[t]{5}{*}{ B } & 7 & Middle & $18-29$ & 4.60 & 0.94 \\
\hline & 8 & Middle & $18-31.5$ & 9.62 & 0.67 \\
\hline & 8 & Base & $31.5-44.5$ & 0.07 & 1.03 \\
\hline & 9 & Middle & $16-28$ & 0.87 & 0.90 \\
\hline & 9 & Base & $28-40$ & 0.01 & 1.12 \\
\hline \multirow[t]{7}{*}{ A } & 10 & - & - & Sandy layer & - \\
\hline & 11 & Top & 9-19 & 8.81 & 0.56 \\
\hline & 11 & Middle & $19-29$ & 0.02 & 0.81 \\
\hline & 12 & Middle & $16-32$ & 9.34 & 0.69 \\
\hline & 12 & Base & $32-47.5$ & 5.96 & 0.73 \\
\hline & 13 & Top & $10-22$ & 7.09 & 0.70 \\
\hline & 13 & Middle & $22-34$ & 1.14 & 1.03 \\
\hline
\end{tabular}

body. The completely contaminated sediment represents $22 \%$ of the area. It was divided into sub-areas A-D in order to facilitate the calculation of the total sediment depot. Three selected sediment cores were taken from each sub-area-according to the data by the echo-sounding profiles and to cover a wide range of organic sediment in each sub-area.

In total, twelve subaquatic sediment cores with a maximum length of $65 \mathrm{~cm}$ were taken from the canal by means of a gravity coring system according to Eijelkamp ( $8 \mathrm{~cm}$ diameter, modified). Each sediment core was divided into a maximum of four parts; the upper sandy layer with TOC contents $\leq$ $0.01 \%$ was abolished. Aliquots of the thoroughly homogenised sediment samples were freeze-dried including the pore water and stored at $4{ }^{\circ} \mathrm{C}$.

For the later calculation of the risk potential, two different geometries of the sediment body were used, one with a trapezoid shape (bottom layers are smaller than the surface layers) and one with a column shape (no difference with depth). The dry mass of each layer was calculated via the dry mass of the sediment material and bulk density of the sediment core multiplied with the integrated area from the mapping. The total calculated sediment masses are given in the supporting information (see Table SI-2).

The TOC content was analysed by means of the evolving $\mathrm{CO}_{2}$ by heating under oxygen at $1000^{\circ} \mathrm{C}$ in a C-Mat (LECO, Mönchengladbach, Germany).

\subsection{Release experiments}

For the short-time release experiment in duplicate, $20 \mathrm{~g}$ wet contaminated sediment from the canal, taken in 2006 (stored under anoxic conditions at $4{ }^{\circ} \mathrm{C}$ in the dark) was eluted with $480 \mathrm{~mL}$ water under oxic (through addition of ascorbic acid) and anoxic conditions in brown glass bottles in the darkness. 
The water was stirred gently to simulate a current flow for resuspension. Water sampling was performed three times, after 1,7 and 42 days with a subsequent replacement of the water. All the water was extracted via a liquid-liquidextraction in triplicate according to Frische et al. (2010) and separately analysed.

\subsection{Chemicals and reagents}

All chemicals, reagents and solvents were purchased from Sigma-Aldrich (Taufkirchen, Germany), Merck (Darmstadt, Germany) and Roth (Karlsruhe, Germany). Solvents were distilled and purity was checked by gas chromatography. All glassware was pre-cleaned with acetone and n-hexane prior to usage.

\subsection{Degradation procedures}

The samples were chemically degraded by a sequential procedure to reveal the extractable and different non-extractable fractions (see Fig. 3). Recoveries were taken from Schwarzbauer et al. (2003) and Heim et al. (2005), determined by spiking experiments on annealed sediments. Details of the method are published in Schwarzbauer et al. (2003) and summarized in Fig. 3. The procedures applied to obtain the different fractions are described briefly in the following:

Extraction (extractable fraction) As a first step prior to chemical degradation, all sediment samples were extracted sequentially with $40 \mathrm{~mL}$ of methanol and mixtures of hexane/acetone
$(1: 1 \mathrm{v} / \mathrm{v})$ by shaking for $24 \mathrm{~h}$ in the dark and ultrasonication at $2 \times 450 \mathrm{~W}$ (Fa. Badelin, Berlin, Germany) in a water bath for $30 \mathrm{~min}$. After centrifugation, the combined crude extract was reduced in volume and following separated by liquid chromatography as described in Schwarzbauer et al. (2003).

Chemical degradation Prior to applying the sequential chemical degradation procedure on sediment samples, a possible formation of analytical artefacts was checked. For this purpose, various reference substances $\left(2,4^{\prime}-\right.$ and $4,4^{\prime}$-DDD, 2,2bis(4-chlorophenyl)acetonitrile - 4,4'-DDCN, 2,2-bis(4chlorophenyl)-1,1-dichloroethene - 4,4'-DDE, 2,2-bis(4chlorophenyl)-1-chloroethene- 4,4'-DDMU and 2,2-bis(4methoxyphenyl)-1,1,1-trichloroethane - 4,4'-MDT) were subjected individually to the chemical degradation procedure but formation of artefacts have not been observed.

Hydrolysis (easily releasable fraction) Pre-extracted sediment samples were mixed with $120 \mathrm{mg}$ of $\mathrm{KOH}$ dissolved in a solution of $0.2 \mathrm{~mL}$ of water and $8 \mathrm{~mL}$ of methanol. The mixture was sealed and heated at $105^{\circ} \mathrm{C}$ for $24 \mathrm{~h}$. After this alkaline hydrolysis procedure, the mixture was filtered, acidified to $\mathrm{pH} 3-5$ with $10 \%$ hydrochloric acid solution and, subsequently, extracted three times with dichloromethane. Thereafter, the extract was dried, reduced in volume to $0.5 \mathrm{~mL}$ and separated into two fractions by column chromatography using dichloromethane (fraction 1) and methanol (fraction 2) as eluent. Prior to GC/MS analysis, $50 \mu \mathrm{L}$ of an internal

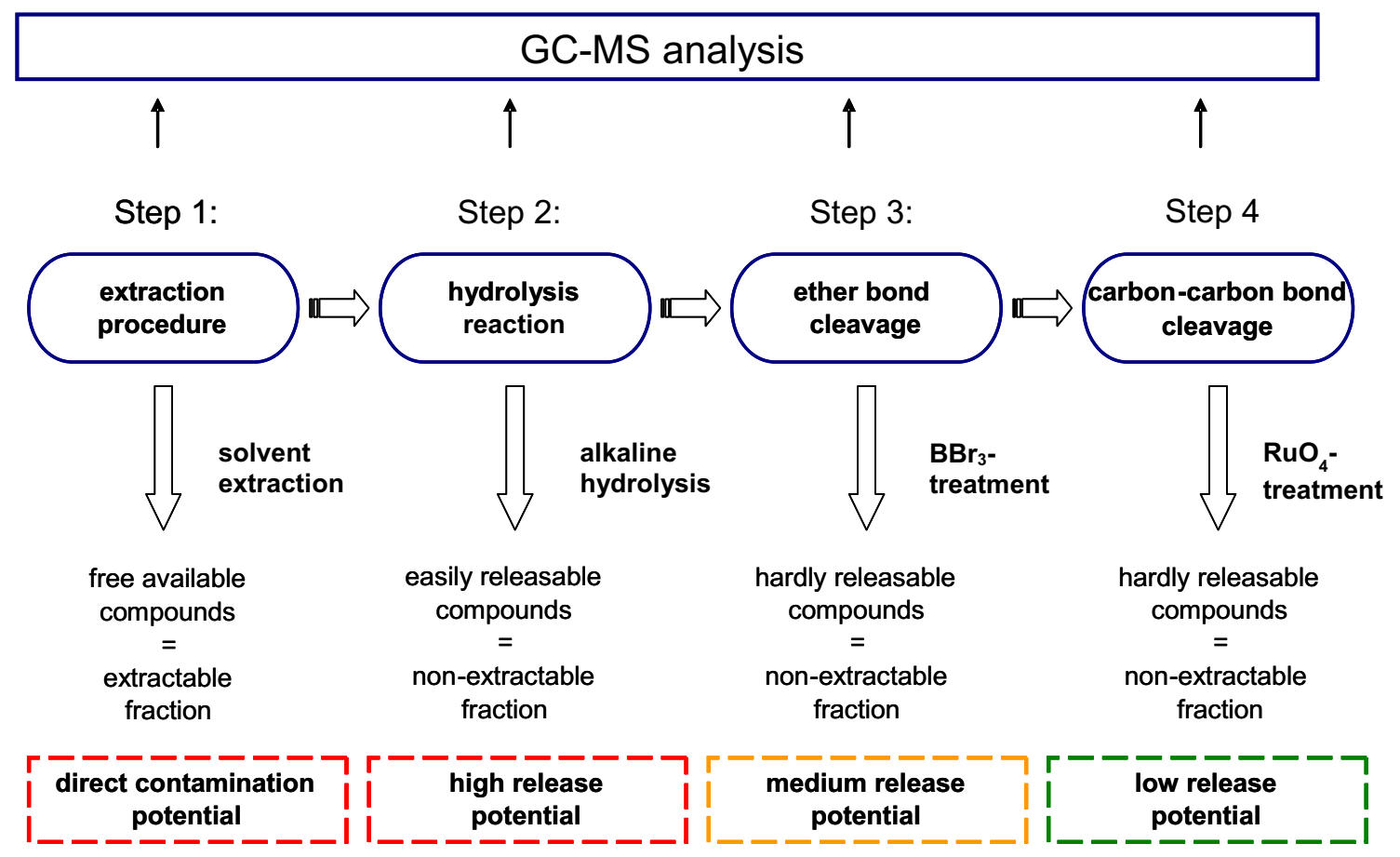

Fig. 3 Scheme of the sequential sediment treatment and the environmental interpretation of the resulting fractions 
standard solution containing $90 \mathrm{ng} \mu \mathrm{L}^{-1} \mathrm{~d}_{34}$-hexadecane was added to each fraction.

$\mathrm{BBr}_{3}$ treatment (hardly releasable fraction) Five millilitres of a 1.0 $\mathrm{M}$ boron tribromide solution in dichloromethane was added to approximately $150 \mathrm{mg}$ of pre-extracted or pre-extracted/saponified dry samples. The extraction procedure followed the procedure for the extractable fraction. After $24 \mathrm{~h}$ of stirring at room temperature, a second ultrasonication step was added. Two milliliters of diethyl ether was added, and the supernatant liquid was decanted and filtered using a Whatman glass fibre filter $(0.7 \mu \mathrm{m}$ pore diameter). The solid residue was washed twice with diethyl ether, and the combined organic layers were washed twice with $5 \mathrm{~mL}$ of preextracted water and dried with anhydrous granulated sodium sulphate. After volume reduction, the crude extracts were separated into three fractions by column chromatography (Baker, $2 \mathrm{~g}$ silica gel $40 \mu \mathrm{m}$ ) using the following eluent mixtures: fraction: 1-n-pentane/dichloromethane $(95 / 5 \mathrm{v} / \mathrm{v})$, fraction 2 : dichloromethane and fraction 3: methanol. Prior to analysis, the extracts were fortified with the same internal standard solution as for the hydrolysis fraction.

$\mathrm{RuO}_{4}$ oxidation (hardly releasable fraction) A mixture of $8 \mathrm{~mL}$ of tetrachloromethane, $8 \mathrm{~mL}$ of acetone and $1 \mathrm{~mL}$ of pre-extracted water was added to aliquots of preextracted or pre-extracted/saponified dry samples and additionally fortified with $5000 \mathrm{mg}$ of sodium periodate and $10 \mathrm{mg}$ of ruthenium tetroxide. The reaction mixture was stirred in darkness for $4 \mathrm{~h}$ and stopped by addition of $50 \mu \mathrm{L}$ of methanol and 2 drops of concentrated sulphuric acid. After decanting the liquid phase, the residue was washed twice with tetrachloromethane. The combined organic layers were collected in a separatory funnel and washed with $5 \mathrm{~mL}$ of pre-extracted water as well as $1 \mathrm{~mL}$ of a sodium thiosulfate solution and subsequently washed with diethyl ether. All organic solutions were combined, dried by means of anhydrous sodium sulphate and reduced to $0.5 \mathrm{~mL}$. The fractionation was realized on the silica gel microcolumns into 2 fractions with fraction 1: dichloromethane and fraction 2: diethyl ether/methanol $(40 / 60 \mathrm{v} / \mathrm{v})$. Prior to GC-MS analysis, the extracts were fortified with the same internal standard solution as for the $\mathrm{BBr}_{3}$-treatment.

\subsection{Analysis by GC-MS}

Gas chromatographic mass spectrometric measurements were performed on a Carlo Erba Mega Series GC 5160 with a $30 \mathrm{~m} \times 0.25 \mathrm{~mm} \times 0.25 \mu \mathrm{m}$ capillary column linked to a Thermoquest Trace Quadrupole-MS. Carrier gas was helium (purity $99.999 \%$ ) set to a velocity of $36 \mathrm{~cm} \mathrm{~s}^{-1}$. The oven temperature was held at $60{ }^{\circ} \mathrm{C}$ for $3 \mathrm{~min}$, then programmed at $5{ }^{\circ} \mathrm{C} \mathrm{min}{ }^{-1}$ to $180^{\circ} \mathrm{C}$ and then programmed at $3{ }^{\circ} \mathrm{C} \mathrm{min}^{-1}$ to $310^{\circ} \mathrm{C}$ and held for $15 \mathrm{~min}$. A $1 \mu \mathrm{L}$ hot spitless injection at $270{ }^{\circ} \mathrm{C}$ was applied with a spitless time of $60 \mathrm{~s}$. The mass spectrometer operated in $\mathrm{EI}^{+}$full scan mode $(70 \mathrm{eV})$ scanning from 35 to $740 \mathrm{~m} / \mathrm{z}$ with a scan time of $0.5 \mathrm{~s}$. Source temperature was set to $250{ }^{\circ} \mathrm{C}$ and interface temperature to $200{ }^{\circ} \mathrm{C}$.

Identification of compounds was based on comparison of gas chromatographic and mass spectral properties of detected substances with those of authentic reference material. The degradation of DDX compounds was checked routinely to be less than $15 \%$ in the injector (US-EPA recommendation). The limit of detection (LOD) was approx. $0.3 \mathrm{ng} \mathrm{g}^{-1}$ with a limit of quantification (LOQ) of $1 \mathrm{ng} \mathrm{g}^{-1}$ d.w. The ion traces recorded are given in the supporting information (see Table SI-1).

\section{Results and discussion}

\subsection{Sediment mapping and raster sampling}

In principle, it was obvious in each of the three profiles that in the area of the uppermost meter, the specific electrical resistances are more or less significantly reduced compared with the deeper subsurface. Whereas at depths of more than $1 \mathrm{~m}$ below the sediment surface specific electrical resistances of more than $65 \Omega \mathrm{m}^{-1}$, mostly more than $95 \Omega \mathrm{m}^{-1}$ were measured, corresponding with the sandy base of the canal. The resistances in the uppermost meter of the sediment layer were mainly at values $<65 \Omega \mathrm{m}^{-1}$, often even below $40 \Omega \mathrm{m}^{-1}$. The specific electrical resistance of the water was $21.3 \Omega \mathrm{m}^{-1}$ at the time of measurement.

Based on the assumption that the sediment in a first approximation is saturated with water of the same temperature and electrical conductivity, the resistances observed in the sediment can be interpreted as follows:

- Areas with an electrical resistivities of more than $65 \Omega \mathrm{m}^{-1}$ are likely to be predominantly affected by sands in natural storage and normal compaction.

- Areas with electrical resistivities below $65 \Omega \mathrm{m}^{-1}$ are categorized as sandy mud with an elevated organic matter and clay mineral content.

The combination of the above two facts points to the conclusion that the near-surface sediment layers were weakly consolidated sediments exhibiting a predominantly sandy matrix with an increased porosity, washing of fine grain into the pore water and an increased content of organic substances in the pore space and the matrix. They were identified with a largest thickness of approximately $1 \mathrm{~m}$ at the western end of the profiles and an average thickness of $0.4 \mathrm{~m}$. 
Based upon these data, sediments with an electrical resistance below $35 \Omega \mathrm{m}^{-1}$ were selected for the sampling of the cores, which are predominantly located in the eastern part of the investigation area, mostly on the northern shore area (see Fig. 4). TOC contents $<0.01 \%$ were not included in the selection. The lithology is presented in Table 1.

The length of the sediment cores (see Table 1) and extrapolation of the dry weight acted as a basis for the calculation of the total sediment mass and the amount of incorporated DDA and relevant DDT metabolites. Calculation of the total sediment body was performed with the two different sediment body geometries, resulting in a total dry sediment mass of $1035 \mathrm{t}$ with the trapezoid approach and $4820 \mathrm{t}$ with the column approach. Details of each core and layer are given in the supporting information (see Table SI-2).

\subsection{Quantification of particle-associated DDT metabolites}

The sequential chemical analysis of the freeze-dried sediment samples (including pore water) revealed a high variety of DDT metabolites that were determined in both non-bound and bound fractions. Noteworthy, the majority of DDT derivatives represents precursor metabolites of DDA (see Fig. 1). The mean content of DDX (as sum of all DDT metabolites) was analysed for the sedimentation area of the canal, subdivided into A-D sections away from the source in area B (see Fig. 4). No differentiation into o, $p^{\prime}$ - and p,p'-DDX was applied. All basic data are given in the supporting information (see Table SI-3).

The DDT metabolites are divided into freely available (EF), easily (ER) and hardly releasable fractions (HR, according to Fig. 3). The individual concentrations in the different samples varied by a factor of up to more than 20,000 in the EF fraction and up to more than $1,000,000$ in the ER fraction within one layer and up to more than 200,000 and $30,000,000$ within one area, pointing to a high heterogeneity. This must be considered for all quantifications.
Comparing the four areas, area $\mathrm{C}$ was comprised by the highest DDX contents and the highest variation of compounds content (see Figs. 5 and 6, and Table SI-3). In the following, the occurrence in the extractable and non-extractable DDT metabolites is discussed separately.

\subsubsection{Extractable fraction}

In almost every sample, DDX were detected, except for DDT, DDE, DDEt, DDA, DDM and DBP in some samples (see Table SI-4). Especially, the parent compound DDT ranged from not detected, through low concentrations about a few hundred nanograms per gram up to $15,000 \mathrm{ng} \mathrm{g}^{-1}$ in the middle layer close to the source, in accordance with earlier investigations (Schwarzbauer et al. 2003). Downstream from the source, the concentrations mostly decrease. The freely available contaminants were dominated by DDD $\left(183,500 \mathrm{ng} \mathrm{g}^{-1}\right)$ and DDMS (52,000 $\mathrm{ng} \mathrm{g}^{-1}$; see Table SI-3). DDA was only detected in low concentrations close to the source and not detected in the areas $\mathrm{C}$ and $\mathrm{D}$. This observation follows strictly the anaerobic metabolic pathway of DDT as described in Fig. 1.

A comparison of the sum DDX in the top, middle and base layer of the areas A-D is given in Fig. 5. The highest concentrations were measured in the base layer of area $\mathrm{A}$, close to the source region. Figure 5 comprises the min and max values and the median on a logarithmic scale. Unfortunately, no top sample of area $\mathrm{B}$ was gained due to sandy material covering the contaminated layers.

\subsubsection{Easy releasable fraction}

The metabolite composition of the free available fractions differs in comparison to the easily releasable fraction obtained by alkaline hydrolysis. Additionally, the composition of the metabolites is highly different in comparison to the free available fraction.

Main metabolites received from hydrolysis reaction are DDA, DDNU, DBP and DDM. In contrast to the free

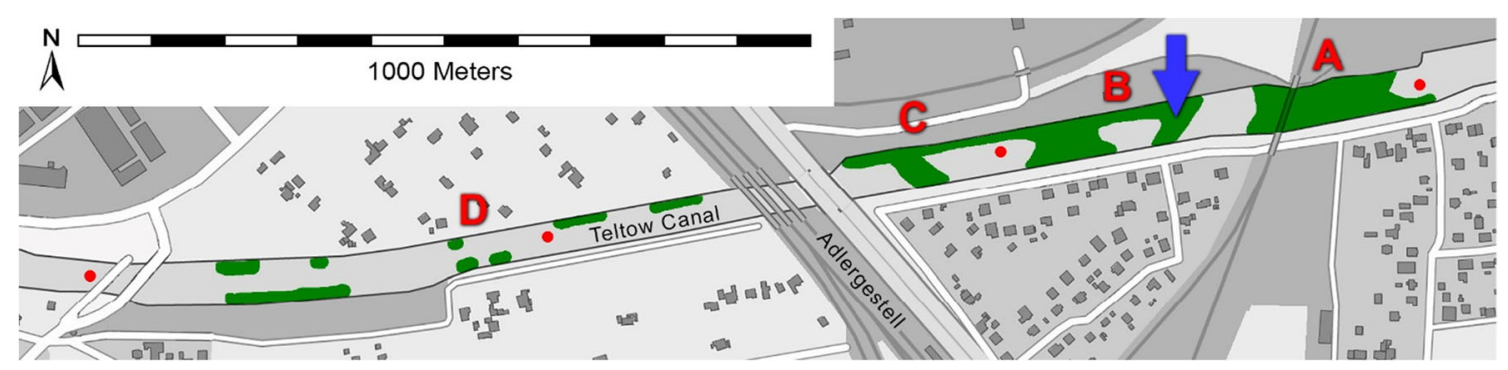

Fig. 4 Results from the sediment field mapping-distinctive sediment areas (green) and the partitioning into A to D with information of the sampling stations of the sediment cores (1 to 13). The blue arrow marks the source-inflow into the Teltow Canal and the red dots are $500 \mathrm{~m}$ indicators. Most of the contaminated sediment was located on the northern riverbank due to the low flow velocity of the canal 
Fig. 5 Comparison of the sum DDX in EF in the layers topmiddle-base from the left to the right $\left(\mathrm{ng} \mathrm{g}^{-1}\right)$. The figure illustrates the highest concentrations in the EF fraction in the base layer of area A, close to the source. Here, the variation was the largest

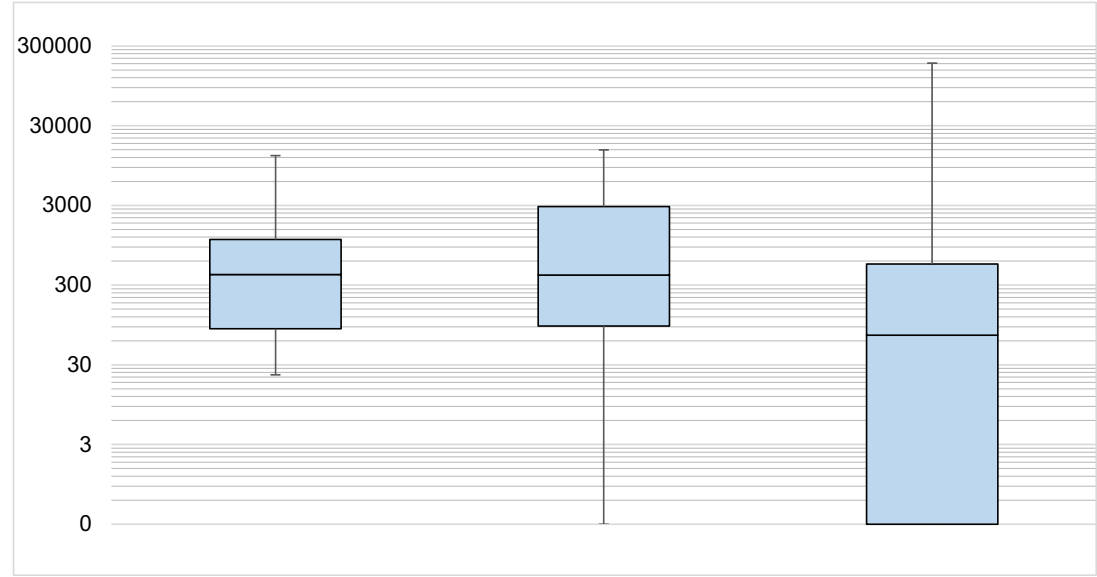

available fraction, these metabolites of the non-extractable residues are dominated by DDA with a mean maximum of $17,000 \mu \mathrm{g} \mathrm{g}^{-1}$ (see Table SI-4). The sample from area $\mathrm{C}$ (middle layer) was characterized by maximum concentrations of DDA (see Table SI-4).

A comparison of the sum DDX in the ER in the top, middle and base layer of the areas A-D is given in Fig. 6. The highest concentrations are measured in area $\mathrm{C}$, close to the source region. The regular flow direction is along the areas $\mathrm{A}$ to $\mathrm{D}$. Analogous to Fig. 5, the min and max values and the median values are presented on a logarithmic scale.

The existence of DDT and metabolites in the investigation area can be explained by the highly reducing environmental conditions (Taylor et al. 2019), for which e.g. a half-life time of up to 65 years has been calculated (Clow et al. 2017). In addition, the decreasing degradation rate due to lesser bioavailability via formation of NER needs to be considered. The contribution of the partially aerobic metabolites to the sum of DDX is in the range of $2-3 \%$ for DDE and up to $28 \%$ in maximum for DDMU in the ER fraction.
As the compounds were introduced as a chemical cocktail, partially with solvent contents in the lower \% degree, the mixture may be preserved. Unfortunately, no information about the emitted number of compounds is available.

For comparison with earlier studies in this investigation area, the date of sampling and the different depths of the sediment cores have to be considered (Schwarzbauer et al. 2003; Heim et al. 2005). In the study presented here, 12 sediment cores represent a wider range of data within the regarded sampling location.

In comparison with reported concentration ranges in other regions, the canal sediments are highly contaminated, partially above those reported for Superfund Sites in the USA (e.g. Garrison et al. 2014; Taylor et al. 2019).

The available Sediment Quality Guidelines (SQG) for freshwater sediments are highly exceeded. Available criteria for freshwater sediments for the preloaded Elbe River System indicate target values of $<5 \mathrm{ng} \mathrm{g}^{-1} \mathrm{~d}$.w. for $\mathrm{p}, \mathrm{p}^{\prime}$-DDT/DDD/ DDE, respectively. The intervention value as the worst case is a factor of 10 higher (Ahlf et al. 2002).
Fig. 6 Comparison of the sum DDX in ER in the top-middle and base layer of areas A-D from the left to the right $\left(\mathrm{ng} \mathrm{g}^{-1}\right)$. The data illustrate the highest concentrations in the middle layer of area $\mathrm{C}$, close to the source. Here, the variation was the largest

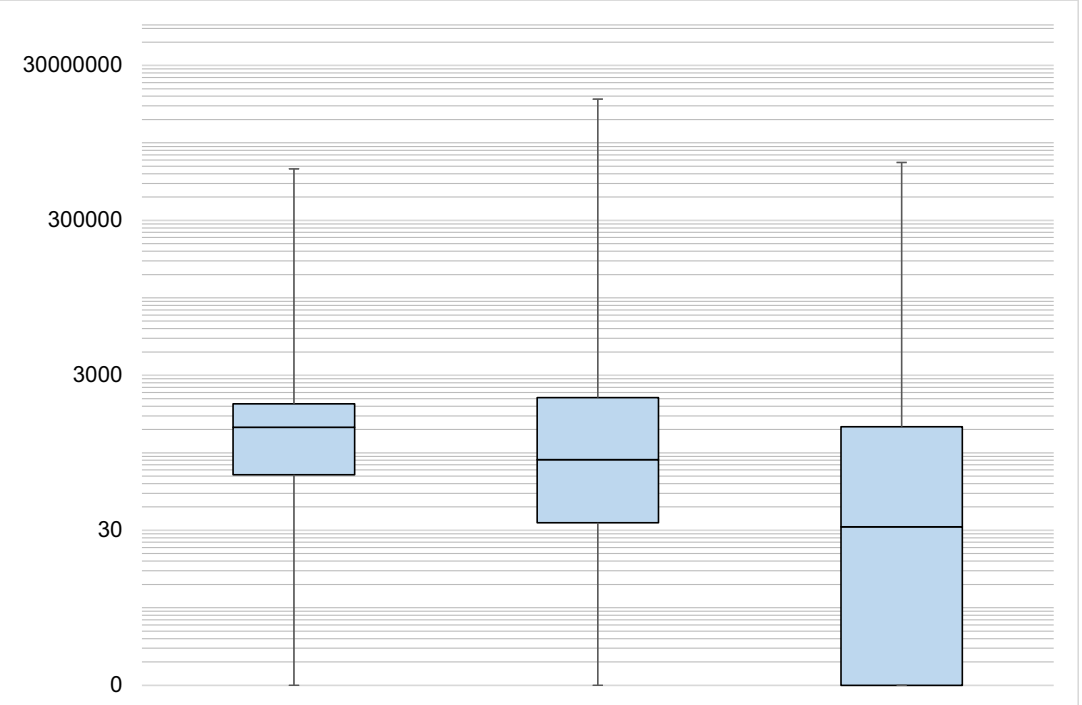


The Canadian SQG states probable effects above $4.77 \mathrm{ng} \mathrm{g}^{-1}$ for $\mathrm{p}, \mathrm{p}^{\prime}$-DDT and $8.51 \mathrm{ng} \mathrm{g}^{-1}$ and $6.75 \mathrm{ng} \mathrm{g}^{-1}$ d.w. for $\mathrm{p}, \mathrm{p}^{\prime}$-DDD and DDE, respectively (Canadian Council of Ministers of the Environment 2002). MacDonald et al. (2000) published a consensusbased SQG TEC for total DDTs (sum of p,p'-DDT/ DDD/DDE) of $5.28 \mathrm{ng} \mathrm{g}^{-1}$ d.w. There are no specific sediment criteria defined and no EQS is available within the WFD. Comparing the top, middle and base sediment layers for each area, the middle layer in area $\mathrm{C}$ reveals the highest contents followed by the base layers, illustrating the complex system of the sediment body (see Figs. 5 and 6). The reason for these varying concentrations in ER may be the different degradation procedure applied and the different sediment type and sampling period, keeping in mind that the formerly cited samples were taken in $1997 / 1999$, potentially consisting of sediment of an undisturbed system in contrast to the sediment sampled in 2007.

The hardly releasable fraction (HR) is obtained by degradation of the pre-hydrolysed sediment with $\mathrm{BBr}_{3}$ and oxidation by $\mathrm{KMnO}_{4}$ or $\mathrm{RuO}_{4}$ (steps 3 and 4; see Fig. 3 ). Cleavage of ether bonds and covalent carbon bond is applied by these operations. The hardly releasable metabolites represent a long-term contamination potential for DDA due to the strong incorporation into the particulate matter or organic matter in the sediment. These NER are not addressed in this paper. Results obtained from $\mathrm{BBr}_{3}$ treatment are lower than in Schwarzbauer et al. (2003). Comparable data for DDA were reported in Schwarzbauer et al. (2003) with a factor 10 higher.

The presented and applied method was compared with newer approaches like pyrolysis-GC-MS and TMAHchemolysis GC-MS (Kronimus et al. 2006; Zhu et al. 2019). Considering potential artefacts, the combination serves as a tool for the assessment of a contamination.

In natural ecosystems, the prevailing sorption of these compounds with a low water solubility occurs on/in finegrained SOM and clay minerals usually coated with humic matter, leading to the formation of NER. Therefore, the accumulation in sediments and organisms leads routinely to the application of a normalisation to the content of total organic carbons (\% TOC), independent of the source, quality and degree of degradation. Often, the $\log K_{\mathrm{oc}}$ is calculated. There was no correlation observed for DDX content and TOC as well as for the contents in the extractable fraction (EF) with the easily releasable fraction (ER). Neither a correlation with the sum of DDX was visible (data not shown).

The metabolism of DDT is proceeded, a long-standing pollution is attested. The semi-final metabolites DDA, DBP, DDCN and DDM are available in noticeable amounts, especially DDA.

\subsection{Calculation of the DDA potential}

As already presented, the DDA contamination potential was calculated using data from precursor metabolites that can be converted to DDA over time and the DDA concentrations themselves. These values have been considered in both the free available and the easily releasable DDA fractions. Hardly releasable proportions have been neglected due to their low potential of a short-time remobilisation.

Further on, a risk for long-term contamination is the existence of the high concentrations of precursor metabolites which can form DDA by metabolism. The very rough estimation is based upon the mean concentrations of each layer. For the base layer in area A, only one concentration was analysed, the data are extrapolated to the other two layers. The estimated DDA release potential shows a wide range of values, given in the brackets as minimum and maximum vales.

\subsubsection{Calculation of the maximum free available amounts of DDA and precursor metabolites}

Data in Table SI-3 were applied to calculate the directly available amounts for the formation of DDA via the precursor metabolites DDT to DDEt. DDA itself was added to the total formation and the final metabolites DDCN to DBP are included in the sum DDX. These were calculated separately obtained by sediment extraction (step 1). They are delivering the percentage of precursors and the direct available percentage of DDA releasable.

The span of the precursors ranges from 88 to $100 \%$ in the EF fraction (see Table SI-4) with a slightly higher formation potential in the middle and deeper layers, considering no data are available for the top layer of area B.

The amounts of calculated maximum DDA contamination are extrapolated by multiplying the maximum concentrations obtained for each sediment area of each layer. The amounts of the labile-bond fraction are directly available (in the case of DDA) or can be released after microbial degradation of precursor metabolites (DDT to DDEt) to DDA.

Summarizing all concentrations up to DDA for all layers and areas, applying the trapezoid model a concentration of $48 \mathrm{~kg}(32-82 \mathrm{~kg})$ results for this fraction. The application of the column model resulted in $278 \mathrm{~kg}(214-354 \mathrm{~kg})$ DDA absolute (see Tables SI-3 and SI-5).

\subsubsection{Calculation of the maximum easily releasable amounts of DDA and precursor metabolites}

Applying the scheme proposed by Zhu et al. (2019), the highest variation occurred in the middle layer of area $\mathrm{C}$ with a factor of more than 10,000 (see Table SI-4). The scheme differentiates the processes into dehalogenation, functional groups formation and loss of one carbon atom for the potential 
precursors down to DDNU. The dehalogenation formation of DDA and metabolites from DDA are included. The release potential is estimated via the progressive release potential and the subsequent formation of metabolites.

The fractions of easily releasable amounts were obtained by basic hydrolysis (step 2). The data of both steps were added for representing the total easily releasable amounts. Especially, in the middle layer of area $\mathrm{C}$, the sum of all precursors plus DDA results in $16,700 \mu \mathrm{g} \mathrm{g}^{-1}$ of the total

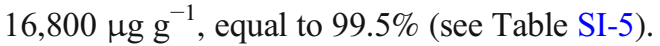

The highest DDA release potential is calculated for the middle layer of area C. Except for the top layers of the areas, $\mathrm{A}$ and $\mathrm{D}$, the data show a release potential between 65 and almost $100 \%$ (see Table SI-4).

\subsubsection{Calculation of the total content and the relative contribution to the DDA contamination range}

Applying the two geometric models for the sediment layers (described in Section 3.1), an extrapolation of the data to the total sediment of $1035 \mathrm{t}$ (trapezoid model) and $4820 \mathrm{t}$ (column model) was realized, being aware of the rough calculation approach. The trapezoid model resulted in $48 \mathrm{~kg}$ DDA formation via precursors for the extractable fraction $(\mathrm{EF})$ and $13.7 \mathrm{~kg}$ via the easy releasable fraction (ER). The direct DDA release potential is $0.4 \mathrm{~kg}$ for the EF and $1346 \mathrm{~kg}$ for the ER (see Table 2), considering the mean contents. The column model resulted in $278 \mathrm{~kg}$ DDA formation via precursors for the extractable fraction (EF) and $47.0 \mathrm{~kg}$ via the easy releasable fraction (ER). The direct DDA release potential is $0.74 \mathrm{~kg}$ for the EF and $9800 \mathrm{~kg}$ for the ER fraction, respectively, considering the mean contents.

The relative contribution of precursors is relatively high in areas $\mathrm{A}$ and $\mathrm{D}$ at the top layer and decreases to almost $1 \%$ in the middle and base layer in the extractable fraction. The calculation excludes the potential DDA degradation products DDCN, DDM and DBP. In the easy releasable fraction, the contribution of DDA is in the middle and base layers around 99\%, except for the top layers in areas $\mathrm{A}$ and $\mathrm{D}$. The highest total contribution is given by the middle layer $\mathrm{C}$ with $75 \%$, depending on the model applied. The relative contribution of DDCN, DDM and

Table 2 Overview of the sum of DDA precursors and DDA direct release content for the areas A-D referring to the applied trapezoid and column model (kg layer ${ }^{-1}$ )

\begin{tabular}{|c|c|c|c|c|c|c|c|c|c|c|c|}
\hline \multicolumn{12}{|l|}{ Trapeziod model } \\
\hline Area & EF & ER & Area & EF & ER & Area & EF & ER & Area & EF & ER \\
\hline $\mathbf{A}$ & Top & Top & B & Top & Top & $\mathbf{C}$ & Top & Top & D & Top & Top \\
\hline Sum DDA precursor & 3.8 & 1.8 & & No sample & No sample & & 1.9 & 1.3 & & 5.1 & 6.1 \\
\hline \multirow[t]{2}{*}{ DDA direct } & 0.0 & 0.4 & & No sample & No sample & & 0.0 & 122.9 & & 0.0 & 2.2 \\
\hline & Middle & Middle & & Middle & Middle & & Middle & Middle & & Middle & Middle \\
\hline Sum DDA precursor & 0.8 & 0.1 & & 0.8 & 0.1 & & 3.0 & 2.3 & & 3.7 & 1.6 \\
\hline \multirow[t]{2}{*}{ DDA direct } & 0.0 & 0.9 & & 0.0 & 1.6 & & 0.0 & 984.7 & & 0.4 & 88.6 \\
\hline & Base & Base & & Base & Base & & Base & Base & & Base & Base \\
\hline Sum DDA precursor & 22.4 & 0.0 & & 0.1 & 0.0 & & 3.7 & 0.4 & & 2.8 & 0.1 \\
\hline DDA direct & 0.0 & 5.6 & & 0.0 & 5.9 & & 0.0 & 130.4 & & 0.0 & 2.7 \\
\hline Sum DDA precursor & 48.1 & 13.7 & & & & & & & & & \\
\hline DDA direct & 0.4 & 1345.9 & & & & & & & & & \\
\hline \multicolumn{12}{|l|}{ Column model } \\
\hline Area & EF & ER & Area & EF & ER & Area & $\mathbf{E F}$ & ER & Area & EF & ER \\
\hline $\mathbf{A}$ & Top & Top & B & Top & Top & $\mathbf{C}$ & Top & Top & D & Top & Top \\
\hline Sum DDA precursor & 9.2 & 2.4 & & No sample & No sample & & 14.5 & 10.1 & & 7.9 & 9.3 \\
\hline \multirow[t]{2}{*}{ DDA direct } & 0.0 & 0.5 & & No sample & No sample & & 0.0 & 940.7 & & 0.0 & 3.3 \\
\hline & Middle & Middle & & Middle & Middle & & Middle & Middle & & Middle & Middle \\
\hline Sum DDA precursor & 0.9 & 0.1 & & 5.7 & 0.9 & & 23.1 & 17.7 & & 5.7 & 2.4 \\
\hline \multirow[t]{2}{*}{ DDA direct } & 0.0 & 1.0 & & 0.0 & 11.7 & & 0.0 & 7534.6 & & 0.6 & 136.4 \\
\hline & Base & Base & & Base & Base & & Base & Base & & Base & Base \\
\hline Sum DDA precursor & 151.3 & 0.0 & & 0.9 & 0.0 & & 32.9 & 3.4 & & 24.9 & 0.6 \\
\hline DDA direct & 0.0 & 37.7 & & 0.0 & 52.2 & & 0.0 & 1166.9 & & 0.1 & 24.7 \\
\hline Sum DDA precursor & 276.9 & 47.0 & & & & & & & & & \\
\hline DDA direct & 0.7 & 9909.7 & & & & & & & & & \\
\hline
\end{tabular}


Fig. 7 Relative contribution of DDA precursors and DDA direct release potential for the ER fraction for the upper, middle and lower layer of areas A to D
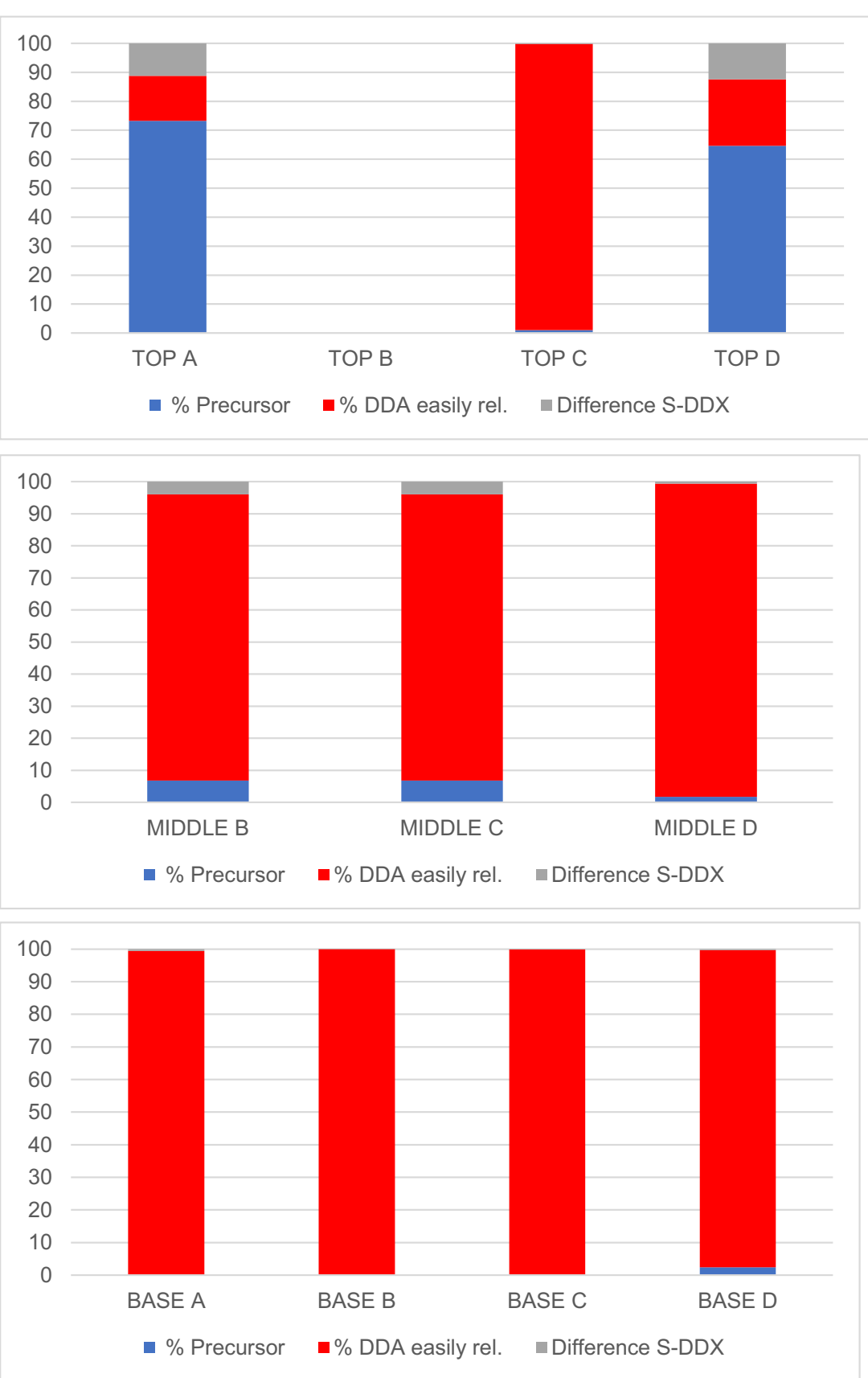

DBP to the sum of DDX is given in Table SI-5 and illustrated in Fig. 7 as difference S-DDX.

The directly available DDA contamination is very low. For the EF fractions, the precursors dominated the contribution. For the ER fraction, the direct available DDA concentration exceeds the formation potential via precursors, except an increased precursor contribution for the top layers in areas A and D.

The main amount of the DDA range is expected by remobilisation of easy-bound amounts through degradation of the SOM or by microbial transformation of free available amounts of precursor metabolites. The easily available DDT metabolites in the ER fraction represent a formation potential of DDA after remobilisation and transformation.

Important is the consideration that the easy releasable fraction can form DDA by degradation processes, providing a huge risk for the aquatic environment. The degradation kinetics are not yet studied in detail; a slow formation of DDA and a subsequent slow further metabolization of DDA is evident. There is almost no contribution of the EF fraction, except for middle layer of area A with a contribution of $22.4 \mathrm{~kg} \mathrm{layer}^{-1}$ and 


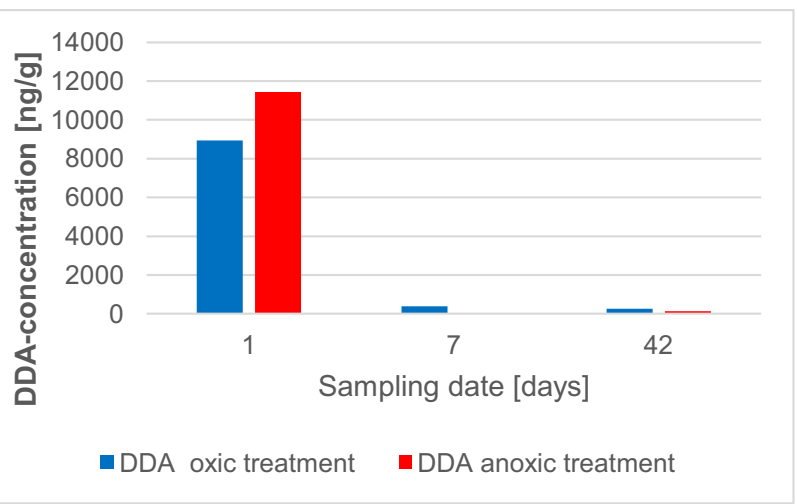

Fig. 8 Remobilisation of DDA from sediment under oxic and anoxic conditions

$150 \mathrm{~kg}$ layer $^{-1}$, for the trapezoid and column model, respectively.

Summarizing the calculations for the different models for the investigation area, the trapezoid model resulted in $48 \mathrm{~kg}$ DDA formation potential in the EF fraction $(32-82 \mathrm{~kg})$ and $1360 \mathrm{~kg}$ in the ER fraction (11-4010 kg). By applying the column model, a $278 \mathrm{~kg}$ DDA formation potential in the EF fraction (214-354 kg) and $9800 \mathrm{~kg}$ in the ER fraction (250$29,000 \mathrm{~kg}$ ) is ascertained. In both cases, the middle layer of area $\mathrm{C}$ contributed around $75 \%$ to the total release potential.

\subsection{Preliminary remobilisation experiments}

Based upon earlier investigations on the sediment contamination, preliminary lab-experiments with real sediment samples were performed to simulate the short-time resuspension within the water body. Especially, the influence of the professional shipping results in dynamic conditions with a potential of resuspension of the upper layers and accordingly building up of a concentration gradient out of the sediment pore water. Heim et al. (2005) published data on the upper most layers of a sediment core clearly illustrating a diffusion gradient out of the sediment into the overlaying water column. Dredging is another opportunity to remobilise sediment layers, only applied exclusively for navigability. The results of the experiments reveal information about the transport of DDA between different environmental compartments. The transport of sedimentary DDA via pore water into the surface water and the transport from water through the soil into the groundwater area are the decisive factors for a possible contamination of the infiltration area of the drinking water plant.

\subsubsection{Release experiment}

Initial laboratory release experiments, simulating the remobilisation/degradation of extractable and nonextractable (NER) residues and the land disposal after dredging in aquaria resulted in a release due to aerobic redox conditions and no differences upon land disposal. Especially, the water phase was rapidly depleted of DDA in the remobilisation scenario with a subsequent adsorption and incorporation into the SPM of the ecosystem.

Under both redox conditions, the maximum of the DDA concentration was released after the first day (93 and 98\%), with no marked differences between oxic and anoxic conditions, indicating that under dynamic conditions, DDA is released by hydrolysis processes (cleavage of the ester bond) and uncoiling of the SOM, independently of the redox potentials (see Fig. 8). An aliquot of the experimental sediment was analysed for DDA, resulting in $24 \mathrm{ng} \mathrm{g}^{-1}$ in the EF fraction and $40,000 \mathrm{ng} \mathrm{g}^{-1}$ in the ER fraction (TOC $8.65 \%$ ). The pore water concentrations were analysed in the range of $160 \mu \mathrm{g} \mathrm{L}^{-1}$. The released DDA concentrations are given in Table 3 .

\section{Conclusions}

The approach offers a tool for the analysis of recent and older contaminations of sediments, including the (bio) available fraction and different fractions from easily to hardly releasable under different environmental conditions.

The different composition of the bound DDT-related compounds shows a high amount of DDA in the hydrolysable ("easily releasable" ER) fraction, in accordance with earlier results (Schwarzbauer et al. 2003). The degradative sediment treatment proves the binding of DDA to the sediment by ester bonds and potential release by hydrolysis reactions.

Summarizing the calculations for the different models within the $1.25 \mathrm{~km}$ long investigation area, the trapezoid model resulted in $48 \mathrm{~kg}$ DDA formation potential in the EF fraction and $1360 \mathrm{~kg}$ in the ER fraction. By applying the column model, a $278 \mathrm{~kg}$ DDA formation potential in the EF fraction and $9800 \mathrm{~kg}$ in the ER fraction is determined. In both cases, the middle layer of area C contributed around $75 \%$ to the total release potential.

The applied degradation scheme allows the determination of the directly releasable contents as well as the determination of the
Table 3 Experimental DDA release from sediment

\begin{tabular}{lll}
\hline Sampling (days) & Oxic treatment DDA $\left(\mathrm{ng} \mathrm{g}^{-1}\right)$ & Anoxic treatment DDA $\left(\mathrm{ng} \mathrm{g}^{-1}\right)$ \\
\hline 1 & 8.938 & 11.442 \\
7 & 385 & 31 \\
42 & 254 & 125 \\
\hline
\end{tabular}


contents that lead to the contaminants by metabolization. Especially, those compounds who tend easily to metabolism (such as p-p'-DDT and p,p'-DMDT) are subjected to degradation and entrapment in the SOM. A further linking via covalent bonds is possible and evaluable with the approach.

The results of the release experiment offer a tool for the first estimation of the environmental behaviour of DDA. The high release behaviour of contaminated sediments for DDA shows that the contamination of water is to be expected, so that DDA must be regarded as a water pollutant.

In the case of dynamic conditions, e.g. while dredging work or by commercial shipping, DDA would be released immediately and in high amounts. Very recent sediment sampling in area B (November 2018) revealed a comparable sandy layer relative to the sampling in 2007. There is no monitoring program performed within the affected canal area water body and sediment.

Acknowledgements We gratefully acknowledge the valuable contributions and discussion of C.G. Bannick (EA Germany) and F. Riedel (FU Berlin) on this manuscript. We would also like to thank O. Menzel and J. Bartels (FU Berlin) for the assistance in sampling and preparation in the lab and J. Evers for graphical support.

Funding Information Open Access funding provided by Projekt DEAL.

Open Access This article is licensed under a Creative Commons Attribution 4.0 International License, which permits use, sharing, adaptation, distribution and reproduction in any medium or format, as long as you give appropriate credit to the original author(s) and the source, provide a link to the Creative Commons licence, and indicate if changes were made. The images or other third party material in this article are included in the article's Creative Commons licence, unless indicated otherwise in a credit line to the material. If material is not included in the article's Creative Commons licence and your intended use is not permitted by statutory regulation or exceeds the permitted use, you will need to obtain permission directly from the copyright holder. To view a copy of this licence, visit http://creativecommons.org/licenses/by/4.0/.

\section{References}

Ahlf W, Hollert H, Neumann-Hensel H, Ricking M (2002) A guidance for the assessment and evaluation of sediment quality, a German approach based on ecotoxicological and chemical measurements. J Soils Sediments 2:1-6

Babut M, Mourier B, Desmet M, Simonnet-Laprade C, Labadie P, Budzinski H, De Alencastro LF, Tu TA, Strady E, Gratiot N (2019) Where has the pollution gone? A survey of organic contaminants in Ho Chi Minh City /Saigon River (Vietnam) bed sediment. Chemosphere 217:261-269

Becker S, Halsall CJ, Tych W, Kallenborn R, Su Y, Hung H (2008) Long-term trends in atmospheric concentrations of $\alpha$ - and $\gamma-\mathrm{HCH}$ in the Arctic provide insight into effects of legislation and climatic fluctuations on contaminant levels. Atmos Environ 42:8233-8255

Bettinetti R, Quadroni S, Boggio E, Galassi S (2016) Recent DDT and PCB contamination in the sediment and biota of Como Bay (Lake Como, Italy). Sci Total Environ 542:404-410

Canadian Council of Ministers of the Environment (2002) Canadian Environmental Quality Guidelines - updated summary Table 2020: http://ceqg-rcqe.ccme.ca/en/index.html
Chen Z, Chen L, Liu Y, Cui L, Tang C-L, Vega H, Krieger RI (2012) Occurrence of DDA in DDT-contaminated sediments of the Southern California Bight. Mar Pollut Bull 64:1300-1308

Clow R, Rutter A, Zeeb BA (2017) Residual dichlorodiphenyltrichloroethane distribution in the soils and sediments of Point Pelee National Park: implications and tools for remediation. Can J Soil Sci 97:178187

de la Cal A, Eljerrat E, Raldúa D, Durán C, Barceló D (2008) Spatial variation of DDT and its metabolites in fish and sediment from Cinca River, a tributary of the Ebro River (Spain). Chemosphere 70:1182-1189

Eganhouse RP, Pontolillo J (2008) DDE in sediments of the Palos Verdes Shelf, California: in situ transformation rates and geochemical fate. Environ Sci Techol 42:6392-6398

Eganhouse RP, Di Filippo E, Pontolillo J, Hackley P, Edwards BD (2018) DDT and related compounds in pore water of shallow sediments on the Palos Verdes Shelf, California, USA. Mar Chem 203:78-90

Ehlers GAC, Loibner AP (2006) Linking organic pollutant (bio) availability with geosorbent properties and biomimic methodology: a review of geosorbent characterisation and (bio)availability prediction. Environ Pollut 141:494-512

Eschenbach A, Kästner M, Bierl R, Schaefer G, Mahro B (1994) Evaluation of a new, effective method to extract polycyclic aromatic hydrocarbons from soil samples. Chemosphere 28:683-692

Eschenbach A, Mescher H, Wienberg R, Mahro B (2001) Humification of PAH and TNT during bioremediation - evaluation of long-term risk and sustainability. In: Stegmann R, Brunner G, Calmano W, Matz G (eds) Treatment of contaminated soil - fundamentals, analysis, applications, pp 271-291

Frische K, Ricking M, Schwarzbauer J (2010) Structural diversity of organochlorine compounds in groundwater affected by an industrial point source. Chemosphere 81:500-508

Garrison AW, Cyterski M, Roberts KD, Burdette D, Williamson J, Avants JK (2014) Occurrences and fate of DDT principal isomers/

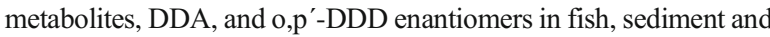
water at a DDT-impacted superfund site. Environ Pollut 94:224-234

Heberer T, Dünnbier U (1999) DDT metabolite bis (chlorophenyl) acetic acid: the neglected environmental contaminant. Environ Sci Techol 33:2346-2351

Heim S, Ricking M, Schwarzbauer J, Littke R (2005) Halogenated compounds in dated sediment cores of the Teltow Canal, Berlin: time related sediment contamination. Chemosphere 61:1427-1438

Kalathoor R, Zeiner M, Schmidt B, Schäffer A, Schwarzbauer J (2015) First evidence for covalent linkage of acidic metabolites of metalaxyl and DDT as non-extractable pesticide residues in soil and sediment. Environ Chem Lett 13:431-437

Kallenborn R (2006) Persistent organic pollutants (POPs) as environmental risk factors in remote high-altitude ecosystems. Ecotoxicol Environ Saf 63:100-107

Kästner M, Nowak KM, Miltner A, Schäffer A (2016) (Multiple) isotope probing approaches to trace the fate of environmental chemicals and the formation of non-extractable "bound" residues. Curr Op Biotech 41:73-82

Kronimus A, Schwarzbauer J, Ricking M (2006) Analysis of nonextractable DDT-related compounds in riverine sediments from the Teltow Canal, Berlin, by pyrolysis and thermochemolysis. Environ Sci Technol 40:5882-5890

Lee H II, Lincoff A, Boese BL, Cole FA, Ferraro SP, Lamberson JO, Ozretich RJ, Randall RC, Rukavina KR, Schults DW, Sercu KA, Specht DT, Swartz RC, Young DR (1994) Ecological risk assessment of the marine sediments at the United Heckathorn superfund site. ERL-N 269:1-498

Luthy RG, Aiken GR, Brusseau ML, Cunningham SD, Gschwend PM, Pignatello JJ, Reinhard M, Traina SJ, Weber WJ Jr, Westall JC (1997) Sequestration of hydrophobic organic contaminants by geosorbents. Environ Sci Technol 31:3341-3347 
MacDonald DD, Ingersoll CG, Berger TA (2000) Development and evaluation of consensus-based sediment quality guidelines for freshwater ecosystems. Arch Environ Contam Toxicol 39:20-31

Marei ASM, Quirke JME, Rinaldi G, Zoro JA, Eglinton G (1978) The environmental fate of DDT. Chemosphere 2:993-998

Mitra S, Corsolini S, Pozo K, Audy O, Sarkar SK, Biawas JK (2019) Characterization, source identification and risk associated with polyaromatic and chlorinated organic contaminants (PAHs, PCBs, PCBzs and OCPs) in surface sediments of Hoogly estuary, India. Chemosphere 221:154-165

Northcott GL, Jones KC (2000) Experimental approaches and analytical techniques for determining organic compound bound residues in soil and sediment. Environ Pollut 108:19-43

Nowak KM, Kästner M, Miltner A, Trapp S, Schäfer A (2014) Nonextractable residues (NER) from xenobiotics in soil: a new classification and relevance in the risk assessment. Science Across Bridges, Borders and Boundaries: Programme Book Basel. SETAC-Europe, Switzerland

Qu C, Albanese S, Lima A, Hope D, Pond P, Fortelli A, Romano N, Cerino P, Pizzolante A, De Vivo B (2019) The occurrence of OCPs, PCBs, and PAHs in the soil, air, and bulk deposition of the Naples metropolitan area, southern Italy: implications for sources and environmental processes. Environ Int 124:89-97

Schäffer A, Kästner M, Trapp S (2018) A unified approach for including non-exzractable residues (NER) of chemicals and pesticides in the assessment of persistence. Environ Sci Eur 30:51

Scheunert I, Schröder P (1998) Formation, characterization and release on non-extractable residues of $\left[{ }^{14} \mathrm{C}\right]$-labeled organic xenobiotics in soils. Environ Sci Pollut Res 5:238-244

Schwarzbauer J, Ricking M, Littke R (2003) DDT-related compounds bound to nonextractable particulate matter in sediments of the Teltow Canal, Germany. Environ Sci Technol 37:488-495

Semple KT, Morriss AWJ, Paton GI (2003) Bioavailability of hydrophobic organic contaminants in soils: fundamental concepts and techniques for analysis. Eur J Soil Sci 54:809-818

Stohlman EF, Smith MI (1945) The isolation of Di (p-chlorophenyl) acetic acid (DDA) from the urine of rabbits poisoned with 2, 2 - bis (p-chlorophenyl)1,1,1-trichloroethane (DDT). Public Health Rep 60:375-379

Takada H, Eganhouse RP (1998) Molecular markers of anthropogenic waste. In: Meyers RA (ed) Encyclopedia of environmental analysis and remediation. Wiley, New York, p 2883-2940

Taylor AR, Wang J, Liao C, Schlenk D, Gan J (2019) Effects of aging on bioaccessibility of DDTs and PCBs in marine sediment. Environ Pollut 246:582-589

Vagi MC, Petsas AS (2020) Recent advances on the removal of priority organochlorine and organophosphorus bio recalcitrant pesticides defined by Directive 2013/39/EU from the environmental matrices by using advanced oxidation processes: an overview (2007-2018). J Environ Chem Eng 8:102940

Wan Y, Hu J, Liu J, An W, Tao S, Jia Z (2005) Fate of DDT-related compounds in Bohai Bay and its adjacent Haihe Basin, North China. Mar Pollut Bull 50:439-445

Wetterauer B, Ricking M, Otte J, Hallare AW, Rastall A, Erdinger L, Schwarzbauer J, Braunbeck T, Holler H (2012) Toxicity and endocrine effects of DDT and its metabolites DDA, DDMU, DDMS and DDCN. Environ Sci Pollut Res 19:403-415

White WC, Sweeny TR (1945) The metabolism of 2,2bis ( $\mathrm{p}$ chlorophenyl) 1,1,1 trichloroethane (DDT): I. A metabolite from rabbit urine, di(p-chlorophenyl) acetic acid; its isolation, identification and synthesis. Public Health Rep 60:66

WHO (World Health Organization) (2011) WHO position on DDT use in disease vector control under the Stockholm Convention on persistent organic pollutants. WHO/HTM/GMP/2011

WSA (2018) http://www.wsa-b.de/

Zhu X, Dsikowitzky L, Kucher S, Ricking M, Schwarzbauer J (2019) Formation and fate of point-source non-extractable DDT-related compounds on their aquatic-terrestrial pathway. Environ Sci Technol 53:1305-1314

Publisher's note Springer Nature remains neutral with regard to jurisdictional claims in published maps and institutional affiliations. 